\title{
Ebola virus disease outbreak in Nigeria: Transmission dynamics and rapid control
}

\author{
C.L. Althaus ${ }^{a}{ }^{*}$, N. Low ${ }^{a}$, E.O. Musa ${ }^{b}$, F. Shuaib ${ }^{c}$, S. Gsteiger ${ }^{a}$ \\ a Institute of Social and Preventive Medicine (ISPM), University of Bern, Bern, Switzerland \\ ${ }^{\mathrm{b}}$ World Health Organization - Nigeria Office, Federal Republic of Nigeria \\ ${ }^{\mathrm{c}}$ Federal Ministry of Health, Federal Republic of Nigeria
}

\section{A R T I C L E I N F O}

\section{Article history:}

Received 14 January 2015

Received in revised form 5 March 2015

Accepted 8 March 2015

Available online 21 March 2015

\section{Keywords:}

Ebola virus disease

Outbreak

Basic reproduction number

Mathematical model

Nigeria

\begin{abstract}
A B S T R A C T
International air travel has already spread Ebola virus disease (EVD) to major cities as part of the unprecedented epidemic that started in Guinea in December 2013. An infected airline passenger arrived in Nigeria on July 20, 2014 and caused an outbreak in Lagos and then Port Harcourt. After a total of 20 reported cases, including 8 deaths, Nigeria was declared EVD free on October 20, 2014. We quantified the impact of early control measures in preventing further spread of EVD in Nigeria and calculated the risk that a single undetected case will cause a new outbreak. We fitted an EVD transmission model to data from the outbreak in Nigeria and estimated the reproduction number of the index case at 9.0 (95\% confidence interval [CI]: 5.2-15.6). We also found that the net reproduction number fell below unity 15 days $(95 \% \mathrm{CI}$ : 11-21 days) after the arrival of the index case. Hence, our study illustrates the time window for successful containment of EVD outbreaks caused by infected air travelers.
\end{abstract}

(C) 2015 The Authors. Published by Elsevier B.V. This is an open access article under the CC BY-NC-ND license (http://creativecommons.org/licenses/by-nc-nd/4.0/).

\section{Introduction}

Air travel allows Ebola virus disease (EVD) to spread internationally (Gomes et al., 2014; Bogoch et al., 2015). Nigeria experienced an outbreak of EVD with the arrival of an infected air traveler at the international airport in Lagos on July 20, 2014 (Shuaib et al., 2014; Fasina et al., 2014). The traveler had been exposed to EVD in Liberia, had symptoms during his journey, and died on July 25, 2014, after being admitted to a private hospital in Lagos. Although authorities responded to the outbreak rapidly, there were an additional 19 EVD cases in Lagos and a large city in the south of Nigeria, Port Harcourt. The World Health Organization (WHO) declared Nigeria EVD free on October 20, 2014, after no new cases had been detected for 42 days (World Health Organization, 2014).

Analyses of data from the EVD outbreak in Nigeria can provide important information about the impact of the sudden introduction of EVD in large cities and on the control measures needed to stop such outbreaks. The basic reproduction number $R_{0}$ is defined as the average number of secondary infections generated by an infectious index case at the beginning of an outbreak (Heffernan et al.,

\footnotetext{
* Corresponding author. Tel.: +41316315640.

E-mail address: christian.althaus@alumni.ethz.ch (C.L. Althaus).
}

2005). The aim of control interventions is to reduce the net reproduction number $R_{t}$ during an outbreak (also called the effective or instantaneous reproduction number) below unity so that the outbreak eventually ends. Studying the change in $R_{t}$ during the course of an outbreak provides useful information on the effectiveness of the control measures that were implemented (Chowell et al., 2004; Althaus, 2014; Camacho et al., 2014).

In this study, we fitted an EVD transmission model to the reported daily numbers of incident cases and deaths during the outbreak in Nigeria. This allowed us to estimate the basic reproduction number $R_{0}$, and to describe how the net reproduction number $R_{t}$ changed after control interventions were implemented. We then compare the risks of an outbreak from a single undetected case in Nigeria and the other West African countries with ongoing EVD transmission.

\section{Methods}

\subsection{Model}

We applied an EVD transmission model that we used to estimate the reproduction number of EVD in Guinea, Sierra Leone and Liberia (Althaus, 2014). EVD transmission follows SEIR (susceptible-exposed-infectious-recovered) dynamics (Fig. 1) and 


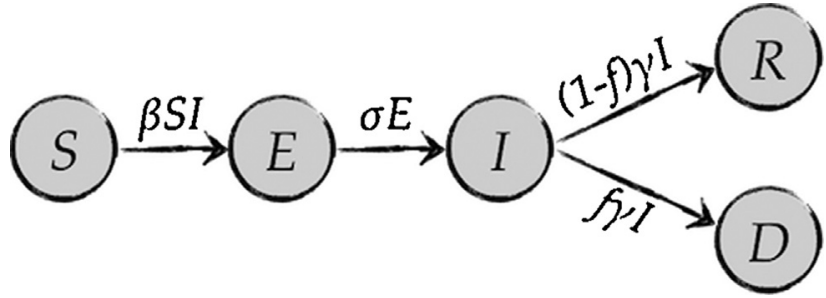

Fig. 1. Schematic illustration of the EVD transmission model. Susceptible individuals $S$ become infected by infectious individuals $I$ at rate $\beta$. They then move through an incubation period $(E)$ at rate $\sigma$ before they become infectious individuals $I$. Infectious individuals $I$ recover or die at rate $\gamma$. The case fatality rate is given by $f$.

can be described by the following set of ordinary differential equations (ODEs):

$$
\begin{aligned}
& \frac{d S}{d t}=-\beta(t) S I, \\
& \frac{d E}{d t}=\beta(t) S I-\sigma E, \\
& \frac{d I}{d t}=\sigma E-\gamma I, \\
& \frac{d R}{d t}=(1-f) \gamma I, \\
& \frac{d D}{d t}=f \gamma I .
\end{aligned}
$$

After infection, susceptible individuals $S$ enter the exposed class $E$ before they become infectious individuals $I$ and either recover $(R)$ or die $(D)$. The average durations of incubation and infectiousness are given by $1 / \sigma$ and $1 / \gamma$, respectively. $f$ is the case fatality rate. The transmission rate before the introduction of control interventions was assumed to be constant, i.e., $\beta(t)=\beta_{0}$. Upon the implementation of control measures at time $\tau$, the transmission rate was assumed to decay exponentially: $\beta(t)=\beta_{0} e^{-k(t-\tau)}$ (Lekone and Finkenstädt, 2006). The basic and net reproduction numbers are given by $R_{0}=\beta_{0} S(0) / \gamma$ and $R_{t}=\beta(t) S(t) / \gamma$, respectively.

We assumed the outbreak started with a single infected case in a large susceptible population $\left(I(0)=1\right.$ and $\left.S(0)=10^{6}\right)$. As long as the number of cases is small compared to the total population size, the exact number of susceptible individuals does not need to be known to estimate the model parameters. The ODEs were solved numerically in the R software environment for statistical computing (Development Core Team, 2014) using the function ode from the package deSolve.

We assumed the observed daily numbers of incident cases and deaths to be Poisson distributed (Nishiura and Chowell, 2014; Camacho et al., 2014; Ebola Response Team, 2014) to derive maximum likelihood estimates (MLEs) of the following model parameters (Bolker, 2008): the baseline transmission rate $\beta_{0}$, the rate $k$ at which control measures reduce transmission, and the case fatality rate $f$. The average durations of incubation $(1 / \sigma)$ and infectiousness $(1 / \gamma)$ were fixed to values obtained from other data sets (2.2). We also set $\tau=3$ days as the implementation of control measures began on July 23, 2014 (Shuaib et al., 2014). We used the optimization algorithm by Nelder \& Mead, which is implemented in the function optim.

We derived simulation based 95\% confidence intervals (CIs) for the model curve making use of the asymptotic normality of MLEs (Mandel, 2013). We also constructed 95\% prediction intervals (PIs) for the cumulative number of cases and deaths. The algorithm was as follows:

1 Simulate $n=10,000$ values, $\theta_{1}, \ldots, \theta_{n} \sim N(\hat{\theta}, \Sigma)$, where $\hat{\theta}$ is the MLE of the unknown model parameters with associated variance-covariance matrix $\Sigma$, using the function rmvnorm from the package mvtnorm.

2 For each $\theta_{i}$, solve the system of ODEs to obtain the model curves for the cumulative number of infected cases and deaths. For each time-point $t$, use the $2.5 \%$ and $97.5 \%$ quantiles from these bootstrap samples to construct the point-wise CIs for the model.

3 For each epidemic trajectory, simulate a vector of daily incident cases from the sampling model, assuming they are Poisson distributed. For each time-point $t$, use the resulting bootstrap sample of the cumulative number of cases to construct the $95 \%$ PI. Proceed similarly for the number of deaths.

\subsection{Data}

Daily incidence of symptom onset and death were derived from the published reports about confirmed $(n=19)$ and probable $(n=1)$ EVD cases (Shuaib et al., 2014; Fasina et al., 2014). We extended the data set from the time of death of the last case to the date that WHO declared Nigeria EVD free (October 20, 2014) with zero counts for the number of incident cases and deaths.

The mean incubation period of EVD was based on the reported cases from the EVD outbreak in Zaire in 1976 (Breman et al., 1978; Breman and Johnson, 2014). We only used the time of symptom onset after person-to-person contact ( $n=109$, range: $2-21$ days). Fitting a gamma distribution to the data resulted in a mean incubation period of 9.31 days (shape: 3.04 ; rate: 0.33 ).

The mean duration of the infectious period of EVD was calculated from the reported cases in the early transmission chain of the outbreak in Guinea. Baize et al. (2014) described the dates of onset of symptoms and death in 17 patients. We assumed that the infectious period was the difference between these two dates (range: 4-17 days). Fitting a gamma distribution to the data resulted in an average infectious period of 7.41 days (shape: 5.29 ; rate: 0.71 ).

\section{Results}

Fitting the transmission model to the data illustrates the variation around the expected number of cases and deaths for a small EVD outbreak, as observed in Nigeria (Fig. 2). The model provides a good description of the cumulative number of deaths. However, the model shows an earlier and slower increase in the cumulative number of cases, compared to the rapid rise in cases that was observed between 8 and 13 days after the arrival of the index case in Lagos. This discrepancy could be a result of stochastic effects or our assumptions about the transmissibility of EVD (see Section 4). The maximum likelihood estimate (MLE) of the baseline transmission rate $\beta_{0}$ was $1.22 \times 10^{-6}$ per individual per day $(95 \% \mathrm{CI}$ : $0.70 \times 10^{-6}-2.10 \times 10^{-6}$ per individual per day). This corresponds to a basic reproduction number $R_{0}=9.01$ (95\% CI: 5.22-15.55). The rate at which control measures reduce transmission was estimated at $k=0.19$ per day $(95 \% \mathrm{CI}: 0.10-0.38$ per day), and the case fatality rate at $f=0.39$ (95\% CI: $0.14-0.71)$.

The Nigerian Federal Ministry of Health, the Lagos State government and international partners activated an Ebola Incident Management Center on July 23, 2014 (Shuaib et al., 2014). Based on our estimates of the baseline transmission rate $\beta_{0}$ and the rate $k$ at which control interventions reduce transmission, we calculated the decrease in the net reproduction number $R_{t}$ following the introduction of control measures that included case isolation, contact tracing and surveillance (Fig. 3). We estimated that $R_{t}$ dropped below unity 15 days (95\% CI: 11-21 days) after the arrival of the index case, that is, 12 days after control measures were implemented. This is about one serial interval after the index case arrived at the airport in Lagos (Ebola Response Team, 2014) and explains the small number of secondary and tertiary cases that was observed in this outbreak (Shuaib et al., 2014; Fasina et al., 2014). 


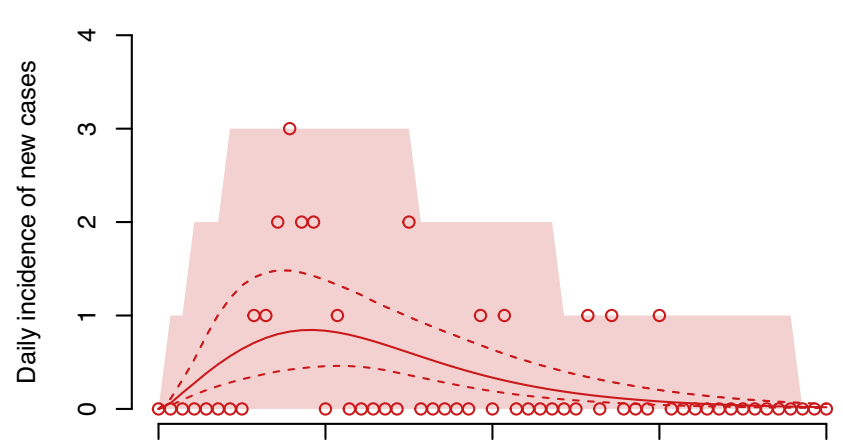

20 Jul 2014 03 Aug $2014 \quad 17$ Aug $2014 \quad 31$ Aug $2014 \quad 14$ Sep 2014

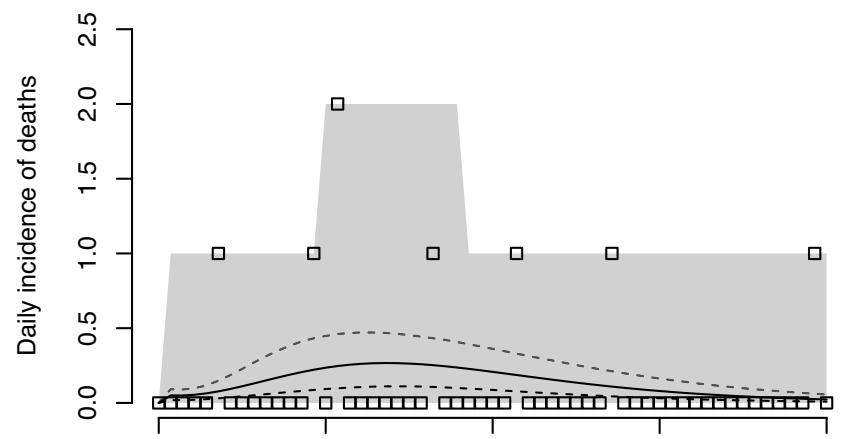

20 Jul $2014 \quad 03$ Aug $2014 \quad 17$ Aug $2014 \quad 31$ Aug $2014 \quad 14$ Sep 2014
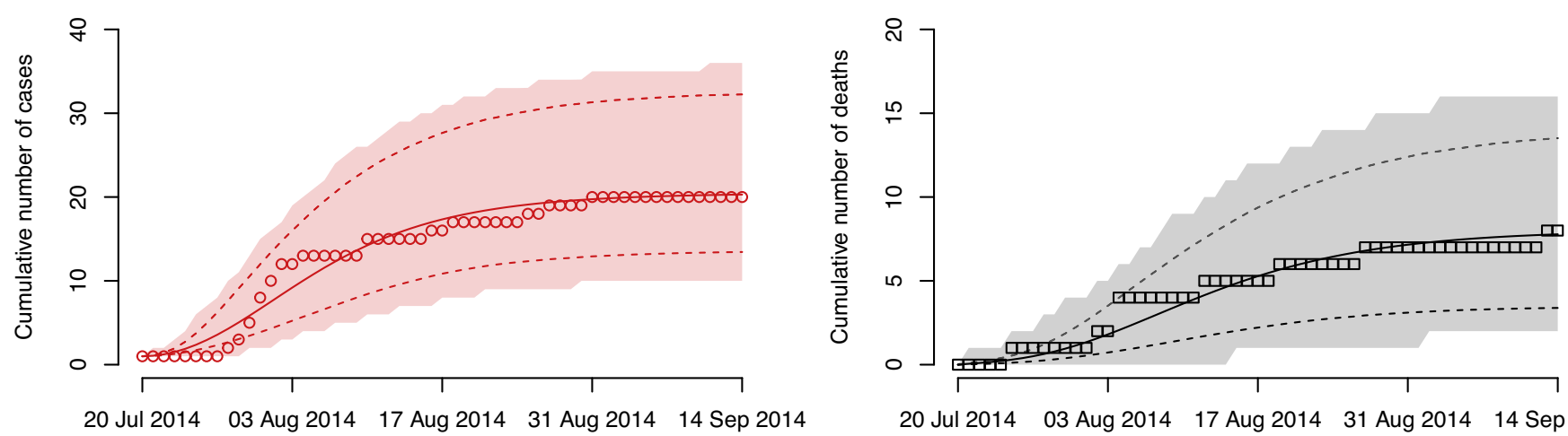

20 Jul $2014 \quad 03$ Aug $2014 \quad 17$ Aug $2014 \quad 31$ Aug $2014 \quad 14$ Sep 2014

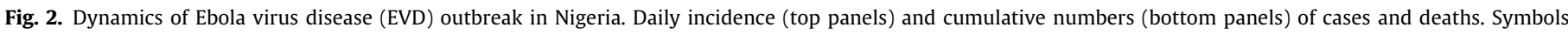

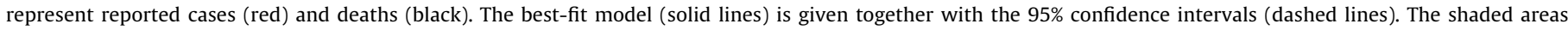
correspond to the $95 \%$ prediction intervals. (For interpretation of the references to color in this figure legend, the reader is referred to the web version of this article.)

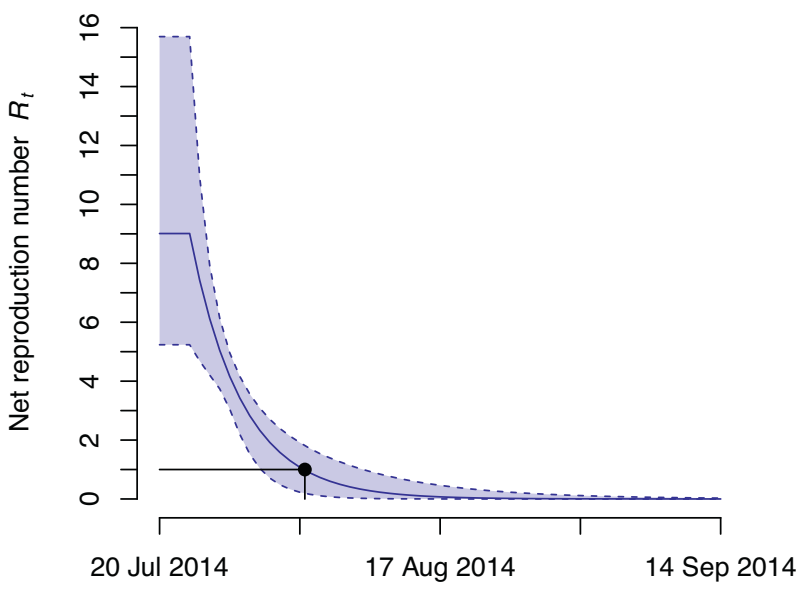

Fig. 3. Net reproduction number $R_{t}$ during the Ebola virus disease (EVD) outbreak in Nigeria. The maximum likelihood estimates of the net reproduction number $R_{t}$ (solid line) are shown together with the 95\% confidence intervals (dashed lines). The black dot denotes the time at which $R_{t}$ dropped below unity (15 days after the arrival of the index case).

Fig. 4 shows the relation between the basic reproduction number $R_{0}$ and the probability $P=1-1 / R_{0}$ that a single infected case that remains undetected causes a subsequent outbreak (Antia et al., 2003). Using the estimated value of $R_{0}$ in Nigeria, we calculated that the risk of an outbreak from a single undetected case was $89 \%(95 \%$ CI: 81-94\%). Our previous estimates of $R_{0}$ for Guinea, Sierra Leone and Liberia are lower than for Nigeria and range between 1.51 and 2.53 (Althaus, 2014). These population-based estimates correspond to outbreak probabilities between $34 \%$ and $60 \%$, which are substantially lower than the risk that we calculated from the reproduction number in Nigeria.

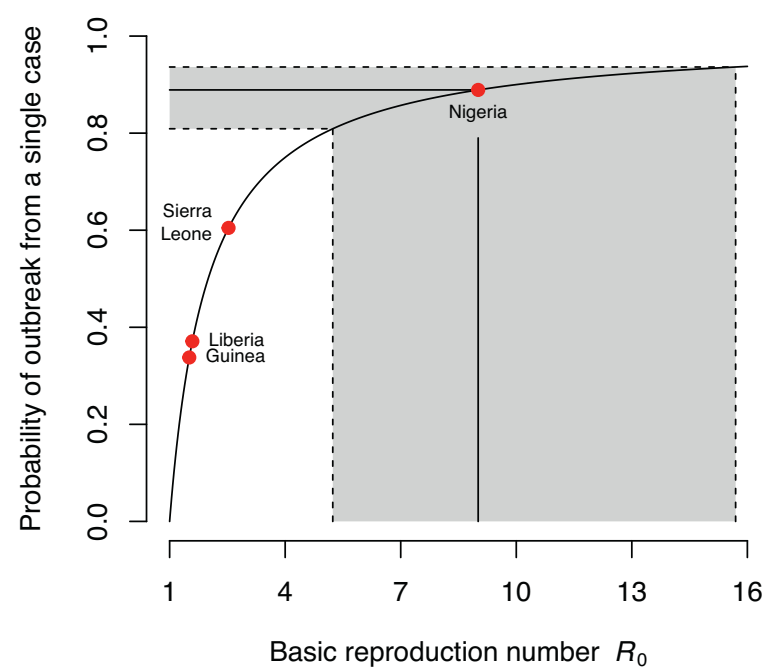

Fig. 4. Risk of an Ebola virus disease (EVD) outbreak from a single undetected case. The curve depicts the relation between the basic reproduction number $R_{0}$ and the probability of an outbreak. The dots correspond to the reproduction number estimated in this study and previously (Althaus, 2014). The shaded area corresponds to the $95 \%$ confidence intervals for Nigeria.

\section{Discussion}

We fitted a dynamic transmission model to data about reported cases and deaths of EVD during a small urban outbreak in Nigeria. We estimated that the basic reproduction number, which can be viewed as an individual reproduction number of the index case, was unusually high $\left(R_{0}=9.01,95 \% \mathrm{CI}: 5.22-15.55\right)$. The rapid implementation of control measures reduced the net reproduction number $R_{t}$ below unity 15 days (95\% CI: 11-21 days) after the arrival of the 
index case. Using the estimated value of $R_{0}$, we calculated that the risk of an outbreak from a single undetected case was $89 \%(95 \% \mathrm{CI}$ : $81 \%-94 \%)$

This study adds to the epidemiological descriptions of the EVD outbreak in Nigeria and benefited from the detailed published data about the reported dates of onset of symptoms and death (Shuaib et al., 2014; Fasina et al., 2014). We estimated $R_{0}$ from the data by extending the modeling approach that provided the first estimates of $R_{0}$ for the 2014 EVD epidemics in Guinea, Sierra Leone and Liberia (Althaus, 2014). Our estimate of the average incubation period (9.3 days), which was based on historical data from the 1976 outbreak in Zaire (Breman et al., 1978), is in good agreement with the observed estimate for single-day day exposures (9.4 days) during the EVD outbreak in West Africa (Ebola Response Team, 2014) and other estimates (Eichner et al., 2011; Chowell and Nishiura, 2014). Similarly, the estimated generation time, the sum of the incubation and infectious periods (16.7 days), is consistent with the serial interval (15.3 days) reported by the Ebola Response Team (2014).

There are several limitations related to the model structure. First, we fitted a deterministic model to a small outbreak of only 20 cases. Stochastic effects could have played an important role in determining the outcome of the outbreak. Fitting a stochastic model, e.g. Gillespie simulations (Gillespie, 1977), to the data would allow us investigate whether the high number of initial transmissions was simply due to chance. Second, we assumed that EVD cases are equally infectious throughout their infectious period. However, transmissibility could increase with disease progression due to higher viral loads (Yamin et al., 2015). This could explain the sudden increase in cases during the second week as most of these were probably infected shortly before the index case died. Third, we did not distinguish between transmission in health-care settings and in the community so we could not examine their separate contributions, as in some other studies (Legrand et al., 2007; Fasina et al., 2014). Fourth, we did not distinguish between different interventions such as case isolation, personal protective equipment for healthcare workers and contact tracing. We considered all control measures together and assumed that their implementation led to an exponential reduction in the transmission rate. Lastly, we treated the two transmission clusters in Lagos and Port Harcourt as a single outbreak and assumed that control measures had the same effect in both places.

Interpreting the estimated $R_{0}$ as an individual reproduction number is consistent with the epidemiological contact data describing 12 (Fasina et al., 2014) or 13 (Shuaib et al., 2014) secondary cases directly linked to the index case and the $95 \% \mathrm{CI}$ (5.22-15.55) includes the observed values. Hence, our modeling approach could be used to quantify the transmission potential of index cases if the number of secondary cases were unknown. The high number of infections during the early phase of the outbreak could be attributed to the setting where they took place: a health facility where people had direct exposure to highly infectious body fluids such as blood. The observed challenges around patient compliance could also have contributed to the high level of transmission seen. In this context, it is worth noting that the decision by the index case to obtain medical care in a private instead of a public health facility might have limited further spread. Socio-economic status in Nigeria is an important determinant of health seeking behavior and prompt access to high quality health services. Thus, it is not surprising that the index case, who was a diplomat, attended a private health facility. Even though there was a strike in government hospitals during the outbreak, it is likely that the index case would still have chosen a private health facility.

Despite the large number of secondary cases, no transmissions occurred while the index case traveled from Liberia to Lagos. Because he provided support to his sister who subsequently died from EVD, it is possible that the index case was aware of his infection and took precautions to minimize contact with other passengers. Deliberate avoidance of contact may also explain why an already sick secondary contact of the index case who evaded contact tracers and traveled by air from Lagos to Port Harcourt, was able to initiate another EVD cluster in Port Harcourt without infecting other airline passengers.

The $R_{0}$ for the outbreak in Nigeria should not be confused with the lower values of around 1.5-2.5 estimated for the countries first affected in 2013/2014 (Althaus, 2014; Fisman et al., 2014; Nishiura and Chowell, 2014; Towers et al., 2014; Ebola Response Team, 2014; Stadler et al., 2014). Those estimates are based on a larger number of cases and represent the average number of secondary cases for a particular country as whole. In contrast, the early phase of the EVD outbreak in Nigeria can be considered a superspreading event (Lloyd-Smith et al., 2005), similar to the funerals that are suspected to have contributed to the early spread of EVD in Sierra Leone (Gire et al., 2014; Stadler et al., 2014; Volz and Pond, 2014). Assuming the number of secondary infections caused by each case is described by a geometric distribution with a mean of 2.0 , the probability that a single individual generates 9 or more secondary cases would be $2.6 \%$. This suggests that the individual reproduction number of the index case in Nigeria is either a rare event, or that the number of secondary infections must be overdispersed. A preliminary analysis of the EVD transmission tree by Faye et al. (2015) indicates that the number of secondary cases follows a negative binomial distribution (unpublished results). In that case, superspreading events such as the one observed for the index case during the EVD outbreak in Nigeria are an expected feature of the individual variation in infectiousness.

The $R_{0}$ in this outbreak in Nigeria shows the transmission potential of an index patient arriving in a major urban area with symptomatic EVD. EVD has also spread in September and October 2014 through international air travel to the USA (World Health Organization, 2014), where two healthcare workers became infected. Patients with EVD also traveled by road to Senegal (World Health Organization, 2014) and Mali (World Health Organization, 2014). In Senegal there were no secondary cases but further cases have occurred in Mali. For the outbreak in Nigeria, we found that the net reproduction number $R_{t}$ dropped below unity 15 days after the arrival of the index case at the international airport in Lagos. This suggests that the number of additional infections will be limited if transmission can be stopped within one serial interval of the infection, and illustrates the time window for successful containment of new EVD outbreaks caused through international travel.

\section{Acknowledgments}

C.L. Althaus was funded by an Ambizione grant and S. Gsteiger by the EpideMMIC (Epidemiology and Mathematical Modelling for Infectious disease Control) project from the Swiss National Science Foundation (grants 136737 and 135654, respectively). The funder had no role in study design, data collection and analysis, decision to publish, or preparation of the manuscript.

\section{References}

Althaus, C.L., 2014. Estimating the reproduction number of Ebola virus (EBOV) during the 2014 outbreak in West Africa. PLoS Curr. 6, http://dx.doi.org/10.1371/ currents.outbreaks.91afb5e0f279e7f29e7056095255b288.

Antia, R., Regoes, R.R., Koella, J.C., Bergstrom, C.T., 2003. The role of evolution in the emergence of infectious diseases. Nature 426, 658-661, http://dx.doi.org/ 10.1038/nature02104

Baize, S., Pannetier, D., Oestereich, L., Rieger, T., Koivogui, L., Magassouba, N., Soropogui, B., Sow, M.S., Keï ta, S., De Clerck, H., Tiffany, A., Dominguez, G., Loua, M., Traoré, A., Kolié, M., Malano, E.R., Heleze, E., Bocquin, A., Mély, S., Raoul, H., Caro, V., Cadar, D., Gabriel, M., Pahlmann, M., Tappe, D., Schmidt-Chanasit, J., Impouma, B., Diallo, A.K., Formenty, P., Van Herp, M., Günther, S., 2014. Emergence of Zaire Ebola virus disease in Guinea. N. Engl. J. Med. 371, 1418-1425, http://dx.doi.org/10.1056/NEJMoa1404505. 
Bogoch, I.I., Creatore, M.I., Cetron, M.S., Brownstein, J.S., Pesik, N., Miniota, J., Tam, T., Hu, W., Nicolucci, A., Ahmed, S., Yoon, J.W., Berry, I., Hay, S.I., Anema, A., Tatem, A.J., MacFadden, D., German, M., Khan, K., 2015. Assessment of the potential for international dissemination of Ebola virus via commercial air travel during the 2014 West African outbreak. Lancet 385, 29-35, http://dx.doi.org/10.1016/S0140-6736(14)61828-6.

Bolker, B.M., 2008. Ecological Models and Data in R. Princeton University Press, Princeton, NJ, ISBN:0691125228.

Breman, J., Piot, P., Johnson, K., White, M., Mbuyi, M., Sureau, P., Heymann, D., Van Nieuwenhove, S., McCormick, J., Ruppol, J., et al., 1978. The epidemiology of Ebola hemorrhagic fever in Zaire, 1976. In: Ebola Virus Haemorrhagic Fever., pp. $103-124$.

Breman, J.G., Johnson, K.M., 2014. Ebola then and now. N. Engl. J. Med. 371, 1663-1666, http://dx.doi.org/10.1056/NEJMp1410540.

Camacho, A., Kucharski, A.J., Funk, S., Breman, J., Piot, P., Edmunds, W.J., 2014 Potential for large outbreaks of Ebola virus disease. Epidemics 9, 70-78, http://dx.doi.org/10.1016/j.epidem.2014.09.003.

Chowell, G., Hengartner, N.W., Castillo-Chavez, C., Fenimore, P.W., Hyman, J.M., 2004. The basic reproductive number of Ebola and the effects of public health measures: the cases of Congo and Uganda. J. Theor. Biol. 229, 119-126, http://dx.doi.org/10.1016/j.jtbi.2004.03.006.

Chowell, G., Nishiura, H., 2014. Transmission dynamics and control of Ebola virus disease (EVD): a review. BMC Med. 12, 196, http://dx.doi.org/ 10.1186/s12916-014-0196-0.

Eichner, M., Dowell, S.F., Firese, N., 2011. Incubation period of Ebola hemorrhagic virus subtype Zaire. Osong Publ. Health Res. Perspect. 2, 3-7, http://dx.doi.org/ 10.1016/j.phrp.2011.04.001.

Fasina, F.O., Shittu, A., Lazarus, D., Tomori, O., Simonsen, L., Viboud, C., Chowell, G., 2014. Transmission dynamics and control of Ebola virus disease outbreak in Nigeria, July to September 2014. Euro Surveill. 19, 20920.

Faye, O., Boëlle, P.Y., Heleze, E., Faye, O., Loucoubar, C., Magassouba, N., Soropogui, B., Keita, S., Gakou, T. Bah, E.H.I. Koivogui, L., Sall, A A Cauchemez, S., 2015. Chains of transmission and control of Ebola virus disease in Conakry, Guinea, in 2014: an observational study. Lancet Infect. Dis., http://dx.doi.org/10.1016/ S1473-3099(14)71075-8.

Fisman, D., Khoo, E., Tuite, A., 2014. Early epidemic dynamics of the West African 2014 Ebola outbreak: estimates derived with a simple twoparameter model. PLoS Curr. 6, http://dx.doi.org/10.1371/currents.outbreaks. 89c0d3783f36958d96ebbae97348d571.

Gillespie, D., 1977. Exact stochastic simulation of coupled chemical reactions. J. Phys. Chem. 81, 2340-2361.

Gire, S.K., Goba, A., Andersen, K.G., Sealfon, R.S.G., Park, D.J., Kanneh, L., Jalloh, S., Momoh, M., Fullah, M., Dudas, G., Wohl, S., Moses, L.M., Yozwiak, N.L., Winnicki, S., Matranga, C.B., Malboeuf, C.M., Qu, J., Gladden, A.D., Schaffner, S.F., Yang, X., Jiang, P.P., Nekoui, M., Colubri, A., Coomber, M.R., Fonnie, M., Moigboi, A., Gbakie, M., Kamara, F.K., Tucker, V., Konuwa, E., Saffa, S., Sellu, J., Jalloh, A.A., Kovoma, A. Koninga, J., Mustapha, I., Kargbo, K., Foday, M., Yillah, M., Kanneh, F., Robert, W., Massally, J.L.B., Chapman, S.B., Bochicchio, J., Murphy, C., Nusbaum, C., Young, S., Birren, B.W., Grant, D.S., Scheiffelin, J.S., Lander, E.S., Happi, C., Gevao, S.M. Gnirke, A., Rambaut, A., Garry, R.F., Khan, S.H., Sabeti, P.C., 2014. Genomic surveillance elucidates Ebola virus origin and transmission during the 2014 outbreak. Science 345, 1369-1372, http://dx.doi.org/10.1126/science.1259657.
Gomes, M.F.C., y Piontti, A.P., Rossi, L., Chao, D., Longini, I., Halloran, M.E., Vespignani, A., 2014. Assessing the international spreading risk associated with the 2014 West African Ebola outbreak. PLoS Curr. 6, http://dx.doi.org/10.1371/currents. outbreaks.cd818f63d40e24aef769dda7df9e0da5.

Heffernan, J.M., Smith, R.J., Wahl, L.M., 2005. Perspectives on the basic reproductive ratio. J. R. Soc. Interface 2, 281-293.

Legrand, J., Grais, R.F., Boelle, P.Y., Valleron, A.J., Flahault, A., 2007. Understanding the dynamics of Ebola epidemics. Epidemiol. Infect. 135, 610-621, http://dx.doi.org/10.1017/S0950268806007217.

Lekone, P.E., Finkenstädt, B.F., 2006. Statistical inference in a stochastic epidemic SEIR model with control intervention: Ebola as a case study. Biometrics 62, 1170-1177, http://dx.doi.org/10.1111/j. 1541-0420.2006.00609.x.

Lloyd-Smith, J.O., Schreiber, S.J., Kopp, P.E., Getz, W.M., 2005. Superspreading and the effect of individual variation on disease emergence. Nature 438, 355-359, http://dx.doi.org/10.1038/nature04153.

Mandel, M., 2013. Simulation-based confidence intervals for functions with complicated derivatives. Am. Stat. 67, 76-81, http://dx.doi.org/10.1080 00031305.2013 .783880$.

Nishiura, H., Chowell, G., 2014. Early transmission dynamics of Ebola virus disease (EVD), West Africa, March to August 2014. Euro Surveill. 19, 36.

R Development Core Team, 2014. R: A Language and Environment for Statistical Computing. R Foundation for Statistical Computing, Vienna, Austria.

Shuaib, F., Gunnala, R., Musa, E.O., Mahoney, F.J., Oguntimehin, O., Nguku, P.M., Nyanti, S.B., Knight, N., Gwarzo, N.S., Idigbe, O., Nasidi, A., Vertefeuille, J.F., 2014 Ebola virus disease outbreak - Nigeria, July-September 2014. MMWR Morb. Mortal Wkly. Rep. 63, 867-872.

Stadler, T., Kühnert, D., Rasmussen, D., du Plessis, L., 2014. Insights into the early epidemic spread of Ebola in Sierra Leone provided by vira sequence data. PLoS Curr. 6, http://dx.doi.org/10.1371/currents.outbreaks. 02bc6d927ecee7bbd33532ec8ba6a25f.

Towers, S., Patterson-Lomba, O., Castillo-Chavez, C., 2014. Temporal variations in the effective reproduction number of the 2014 West Africa Ebola outbreak. PLoS Curr. 6, http://dx.doi.org/10.1371/currents.outbreaks. 9e4c4294ec8ce1adad283172b16bc908.

Volz, E., Pond, S., 2014. Phylodynamic analysis of Ebola virus in the 2014 Sierra Leone epidemic. PLoS Curr. 6, http://dx.doi.org/10.1371/currents.outbreaks. 6f7025f1271821d4c815385b08f5f80e.

WHO Ebola Response Team, 2014. Ebola virus disease in West Africa-the first 9 months of the epidemic and forward projections. N. Engl. J. Med. 371, 1481-1495, http://dx.doi.org/10.1056/NEJMoa1411100.

World Health Organization, 2014. Ebola Virus Disease - United States of America http://www.who.int/csr/don/01-october-2014-ebola/en/

World Health Organization, 2014. Mali Confirms Its First Case of Ebola. http://www.who.int/mediacentre/news/ebola/24-october-2014/en/

World Health Organization, 2014. Nigeria is Now Free of Ebola Virus Transmission. http://www.who.int/mediacentre/news/ebola/20-october-2014/en/

World Health Organization, 2014. The Outbreak of Ebola Virus Disease in Senegal is Over. http://www.who.int/mediacentre/news/ebola/17-october-2014/en/

Yamin, D., Gertler, S., Ndeffo-Mbah, M.L., Skrip, L.A., Fallah, M., Nyenswah, T.G., Altice F.L., Galvani, A.P., 2015. Effect of Ebola progression on transmission and contro in Liberia. Ann. Intern. Med. 162, 11-17, http://dx.doi.org/10.7326/M14-2255. 